Supporting information for

\title{
Method for Structural Determination of Lipid-derived Radicals
}

Yuta Matsuoka, ${ }^{\dagger,}$ Yoshihiro Izumi, $₫, \S$ Masatomo Takahashi, ${ }^{\S}$ Takeshi Bamba, $₫, \S$ and Ken-ichi Yamada ${ }^{*} \dagger, \dagger$

†Physical Chemistry for Life Science Laboratory, Faculty of Pharmaceutical Sciences, Kyushu University, 3-1-1 Maidashi, Higashi-ku, Fukuoka, 812-8582, Japan

†AMED-CREST, Japan Agency for Medical Research and Development, 1-7-1 Ohtemachi, Chiyoda-ku, Tokyo, 100-0004, Japan

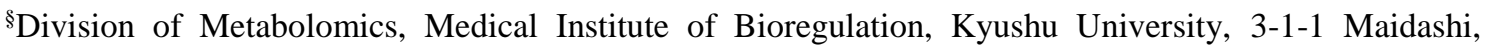
Higashi-ku, Fukuoka, 812-8582, Japan

*Correspondence should be addressed to: Ken-ichi Yamada

E-mail: kenyamada@phar.kyushu-u.ac.jp

\section{Table of Contents}

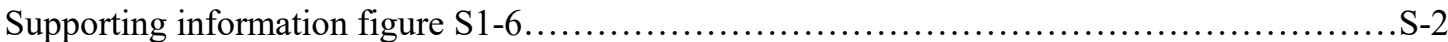

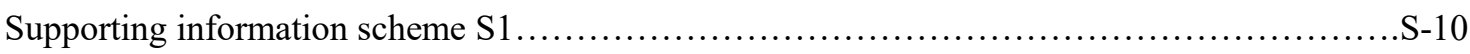

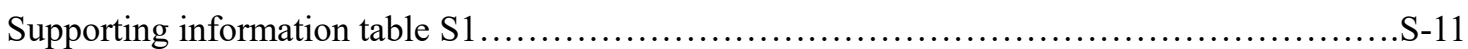

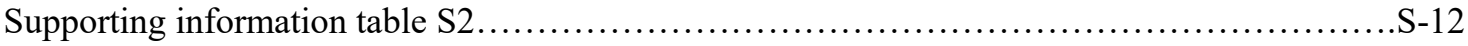

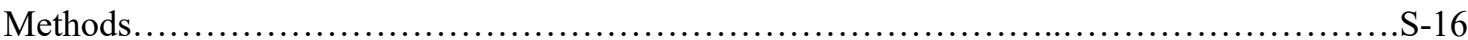

Reagents

Fluorescent standard curve using methoxyamine form of NBD-Pen (NBD-Pen-NOMe)

AAPH-induced generation system in 5\% O2-saturated or Ar-saturated PBS solution Statistical analysis

Supplemental reference. 


\section{Supporting information Figure S1}

a

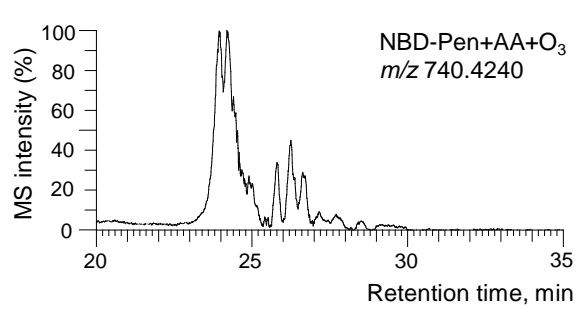

d

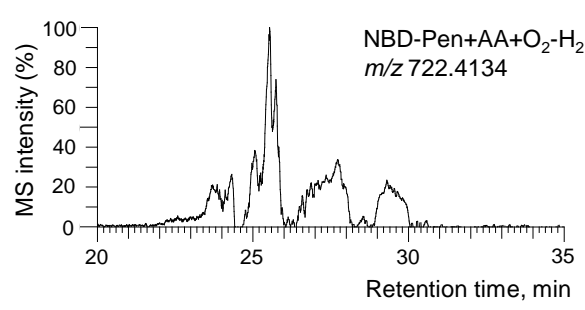

b

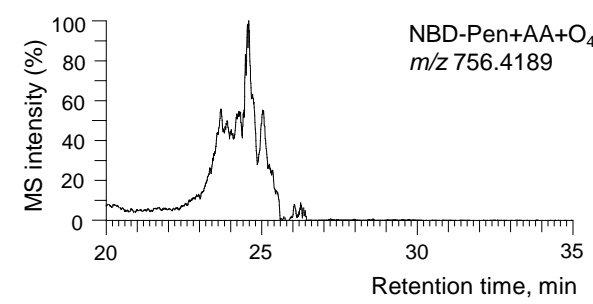

e

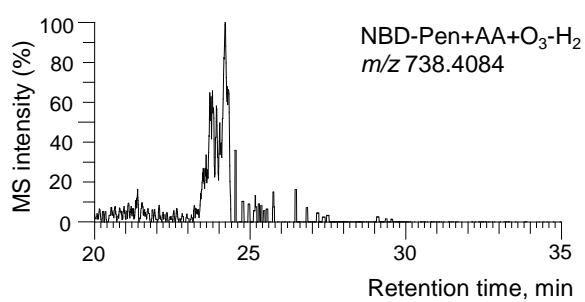

C

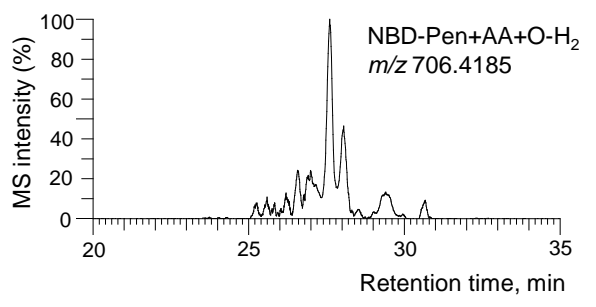

f

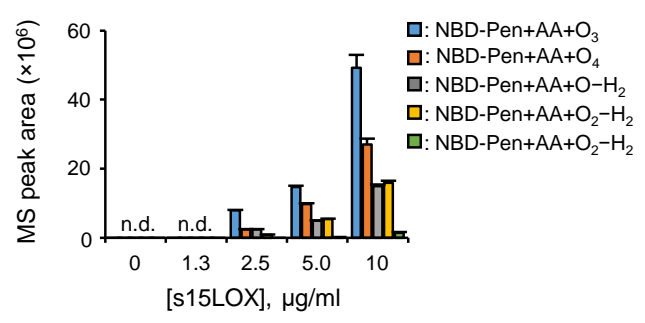

Figure S1 | Extracted ion chromatograms (EICs) (a-e) and semi-quantified data (f) of soybean lipoxygenase from Glycine $\max (\mathrm{s} 15 \mathrm{LOX}$ )-induced lipid-derived radicals. The experimental conditions were the same as described for Figure 1c and 1f-h. Briefly, $500 \mu \mathrm{M}$ arachidonic acid (AA) was stimulated by $10 \mu \mathrm{g} / \mathrm{ml}$ s15LOX with $5.0 \mu \mathrm{M}$ 4-(4nitrobenzo[1,2,5] oxadiazol-7-ylamino)-6-pentyl-2,2,6-trimethylpiperidine-1-oxyl (NBD-Pen) solution (phosphate buffered saline (PBS) in $0.5 \%$ acetonitrile, $\mathrm{pH} 7.4$ ). 


\section{Supporting information Figure S2}

a

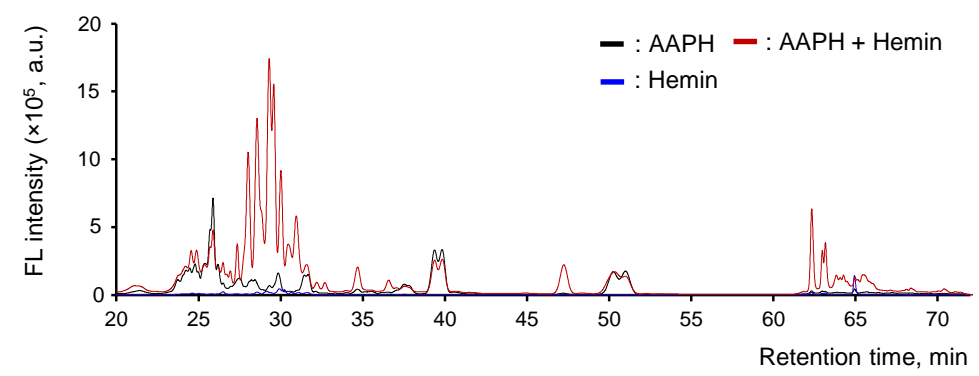

b

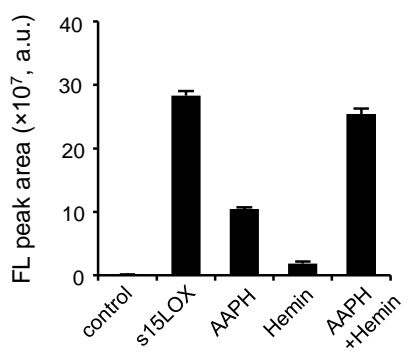

d HRMS spectrum at $47.3 \mathrm{~min}$

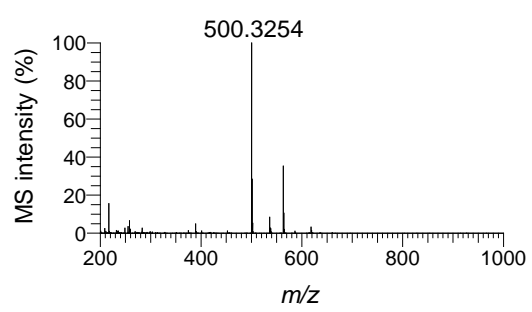

EIC of $m / z 500.3242$

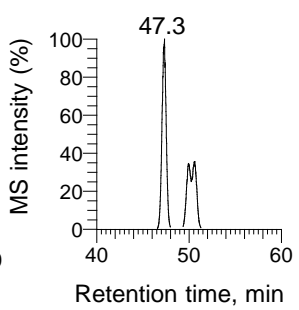

EIC of $m / z 540.3555$

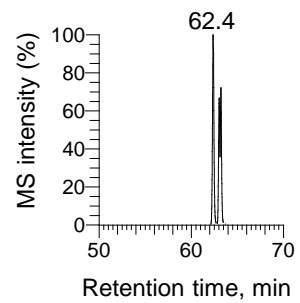

h

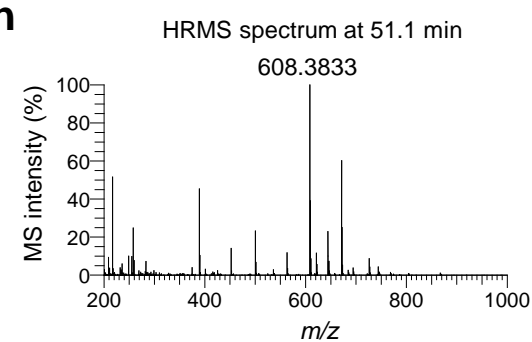

EIC of $m / z 608.3817$

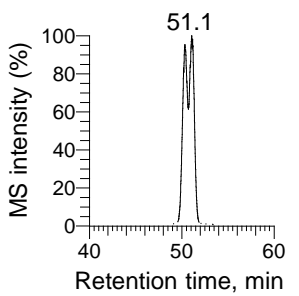

Figure S2 | Detection of lipid-derived truncated radicals. (a) Fluorescence chromatogram and (b) fluorescence peak area of NBD-Pen and lipid-derived radical adducts under different initiation conditions. The experimental conditions were $500 \mu \mathrm{M}$ AA, $5.0 \mu \mathrm{M}$ NBD-Pen, and $10 \mu \mathrm{g} / \mathrm{ml} \mathrm{s} 15 \mathrm{LOX}, 50 \mathrm{mM}$ 2,2'-azobis(2-methylpropionamidine) (AAPH) , $10 \mu \mathrm{M}$ Hemin, or 50 $\mathrm{mM}$ AAPH $+10 \mu \mathrm{M}$ Hemin. (c-h) high-resolution tandem mass spectrometry (HRMS) spectra and extracted ion chromatograms (EICs) of reaction adducts between NBD-Pen and truncated radical adducts, $(\mathbf{c}) \cdot \mathrm{C}_{5} \mathrm{H}_{11},(\mathbf{d}) \cdot \mathrm{C}_{8} \mathrm{H}_{15},(\mathbf{e})$ $\cdot \mathrm{C}_{9} \mathrm{H}_{15} \mathrm{O}$, and $(\mathbf{f}) \cdot \mathrm{C}_{11} \mathrm{H}_{19},(\mathbf{g}) \cdot \mathrm{C}_{12} \mathrm{H}_{19} \mathrm{O}$ and $(\mathbf{h}) \cdot \mathrm{C}_{15} \mathrm{H}_{23} \mathrm{O}$. 


\section{Supporting information Figure S3}

a
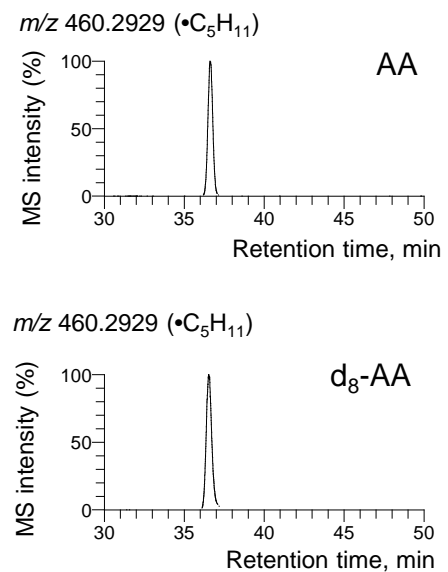

C

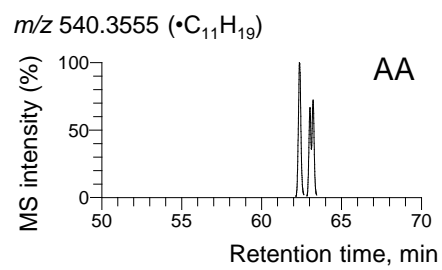

$m / z 544.3807\left(\cdot \mathrm{C}_{11} \mathrm{H}_{15} \mathrm{D}_{4}\right)$

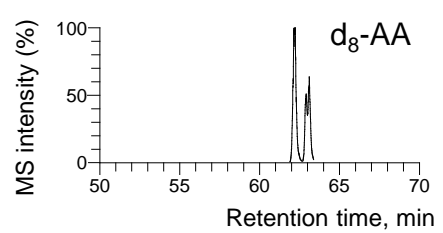

e

$\mathrm{m} / \mathrm{z} 608.3817\left(\cdot \mathrm{C}_{15} \mathrm{H}_{23} \mathrm{O}\right)$

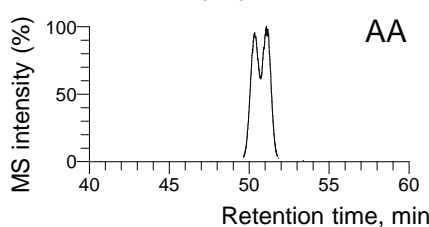

$m / z 615.4258\left(\bullet \mathrm{C}_{15} \mathrm{H}_{16} \mathrm{D}_{7} \mathrm{O}\right)$

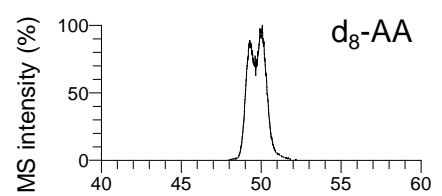

Retention time, min
HRMS/MS spectrum

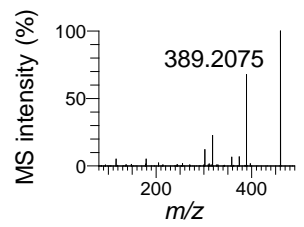

HRMS/MS spectrum

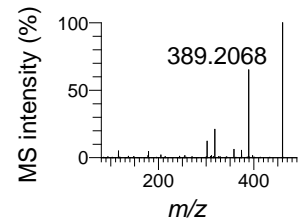

HRMS/MS spectrum

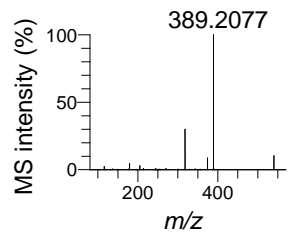

HRMS/MS spectrum

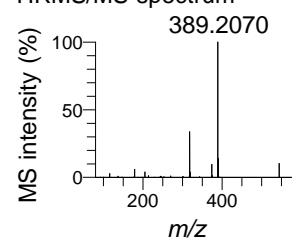

HRMS/MS spectrum

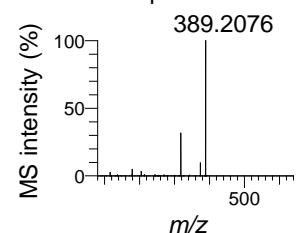

HRMS/MS spectrum

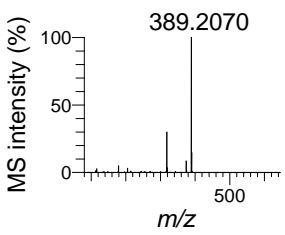

b

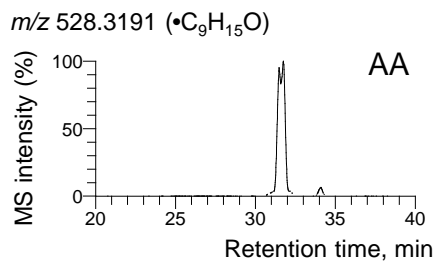

HRMS/MS spectrum
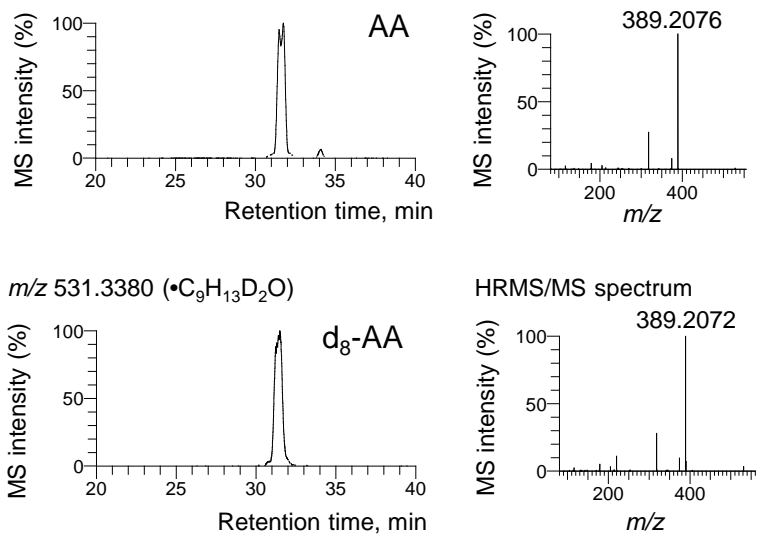

HRMS/MS spectrum

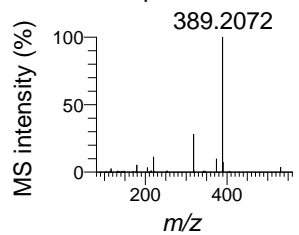

\section{d}

$m / z 568.3504\left(\cdot \mathrm{C}_{12} \mathrm{H}_{19} \mathrm{O}\right)$

HRMS/MS spectrum

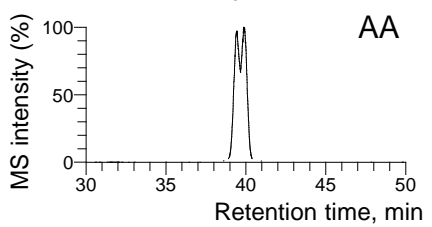

$m / z 573.3819\left(\cdot \mathrm{C}_{12} \mathrm{H}_{14} \mathrm{D}_{5} \mathrm{O}\right)$

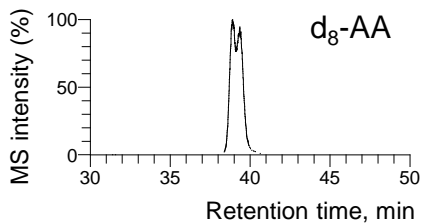

f

$m / z 474.2722\left(\cdot \mathrm{C}_{5} \mathrm{H}_{9} \mathrm{O}\right)$
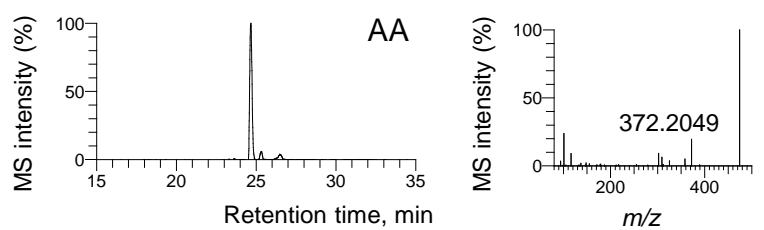

$m / z 474.2722\left(\cdot \mathrm{C}_{5} \mathrm{H}_{9} \mathrm{O}\right)$

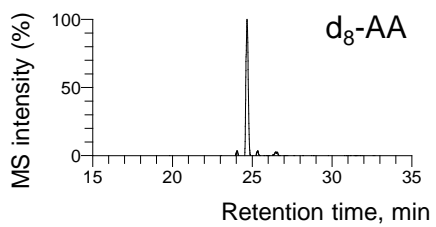

HRMS/MS spectrum

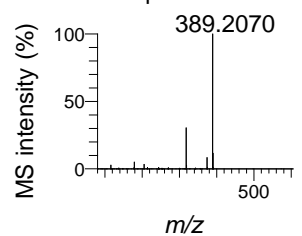

HRMS/MS spectrum

HRMS/MS spectrum
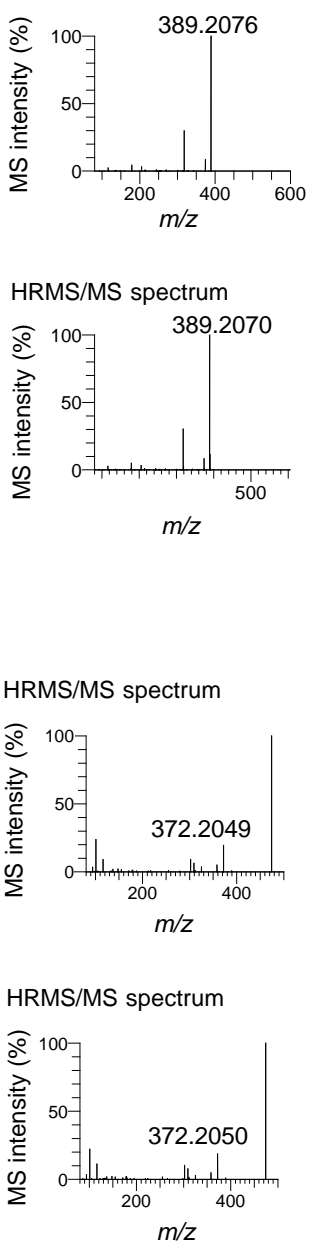
g

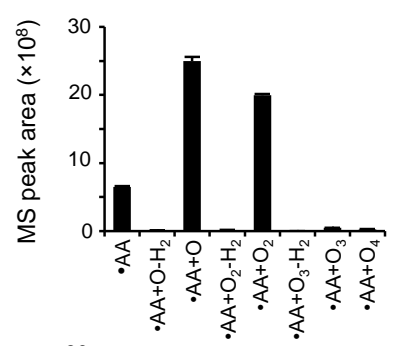

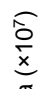
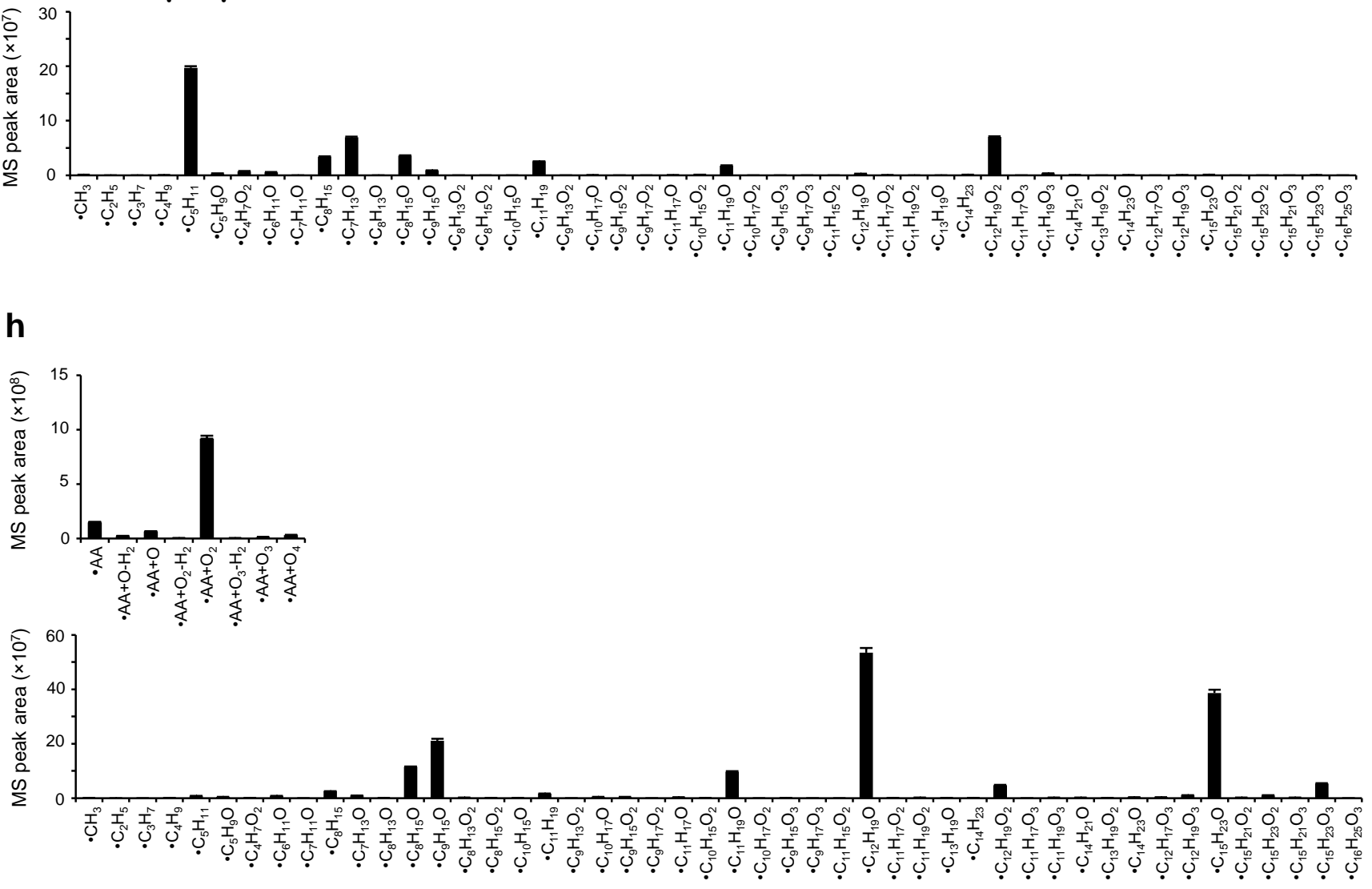


\section{Continued Supporting information Figure S3}
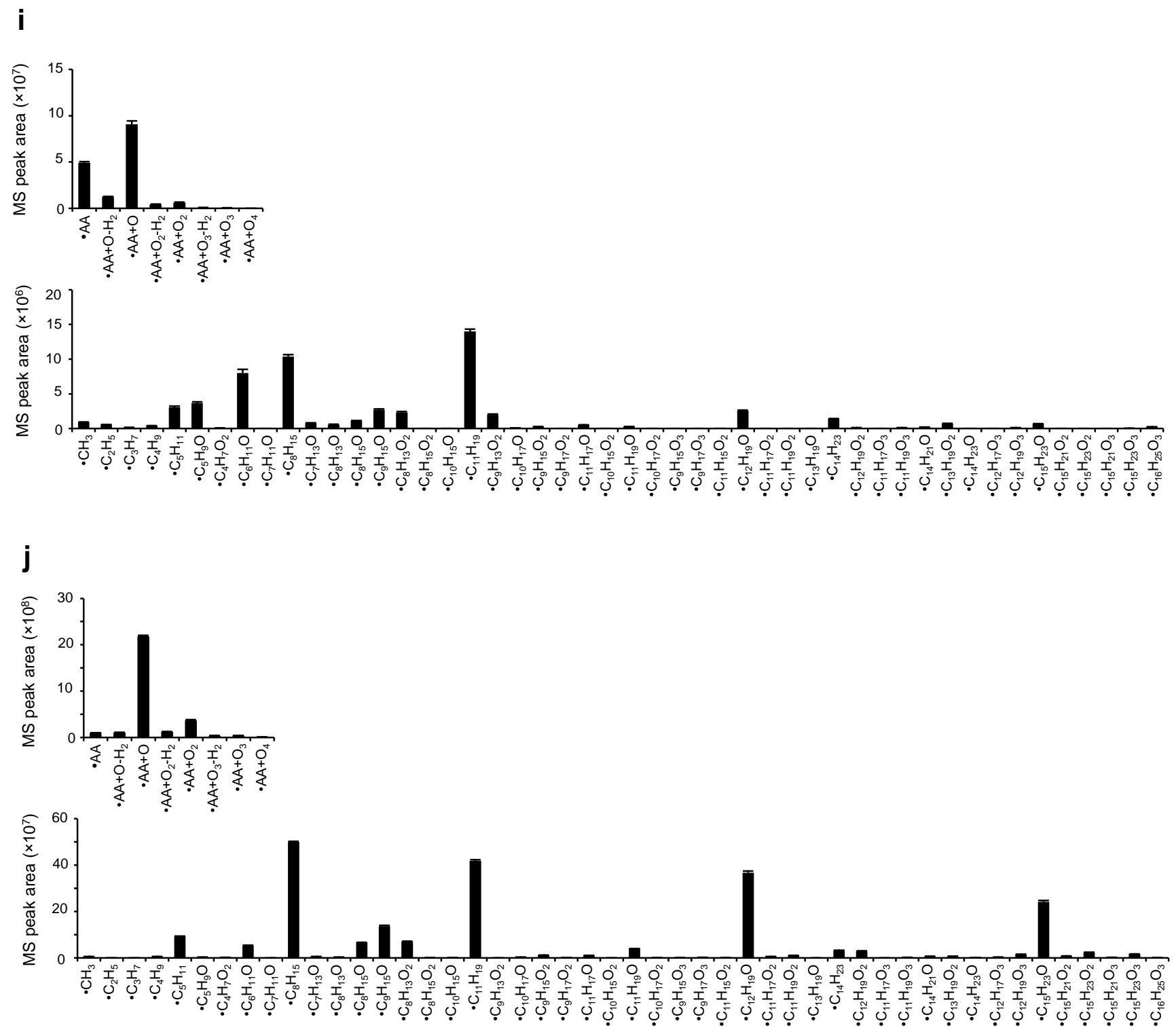

Figure S3 Effect of deuterated AA $\left(d_{8}-\mathrm{AA}\right)$ and stimulant on the EICs of the reaction adducts between NBD-Pen and lipid-derived radicals and these HRMS/MS spectrums. (a-f) AA or 5Z,8Z,11Z,14Z-eicosatetraenoic-5,6,8,9,11,12,14,15- $\mathrm{d}_{8}$ acid, $\left(d_{8}\right.$-AA) $(500 \mu \mathrm{M})$ were used as the substrates with $50 \mathrm{mM} \mathrm{AAPH}$. (a) $\bullet \mathrm{C}_{5} \mathrm{H}_{11},(\mathbf{b}) \cdot \mathrm{C}_{9} \mathrm{H}_{15} \mathrm{O}$ and $\bullet \mathrm{C}_{9} \mathrm{H}_{13} \mathrm{D}_{2} \mathrm{O},(\mathbf{c})$ $\cdot \mathrm{C}_{11} \mathrm{H}_{19}$ and $\bullet \mathrm{C}_{11} \mathrm{H}_{15} \mathrm{D}_{4},(\mathbf{d}) \cdot \mathrm{C}_{12} \mathrm{H}_{19} \mathrm{O}$ and $\bullet \mathrm{C}_{12} \mathrm{H}_{14} \mathrm{D}_{5} \mathrm{O}$, (e) $\bullet \mathrm{C}_{15} \mathrm{H}_{23} \mathrm{O}$ and $\bullet \mathrm{C}_{15} \mathrm{H}_{16} \mathrm{D}_{7} \mathrm{O}$, (f) $\bullet \mathrm{C}_{5} \mathrm{H}_{9} \mathrm{O}$. The reaction conditions are as described for Fig. 2b. (g-j) Original data for Fig. 2e. The experimental conditions were the same as described in Fig.2e. Data are mean \pm s.d. and experiments were performed in triplicate. 


\section{Supporting information Figure S4}
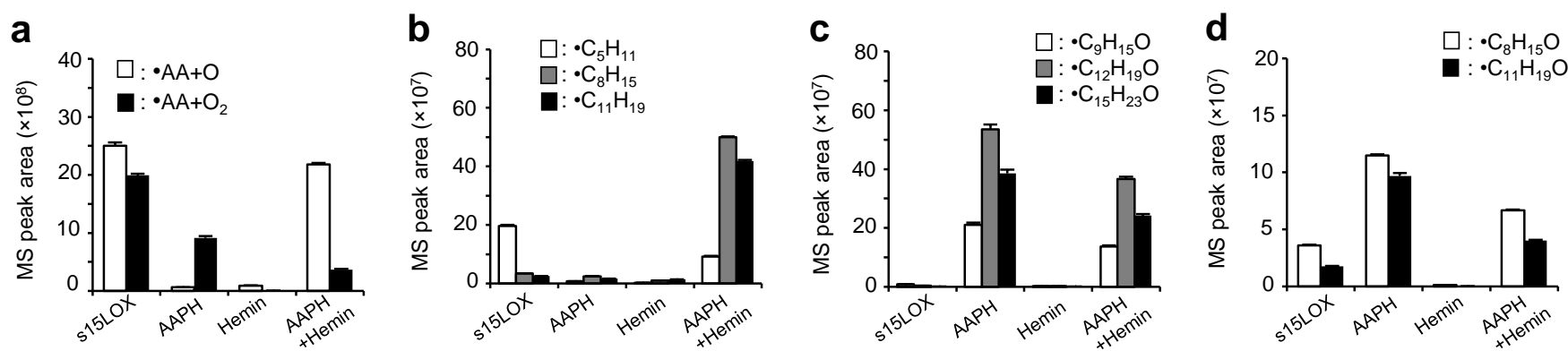

Figure S4 | Semi-quantified lipid-derived radical products stimulated by different radical initiators. (a) Major full-length radicals, (b) $\beta$-scission products, (c) Hock or dioxetane cleavage products, (d) oxygenated truncated products. The experimental conditions were $500 \mu \mathrm{M}$ AA and $5.0 \mu \mathrm{M}$ NBD-Pen, and $10 \mu \mathrm{g} / \mathrm{ml} \mathrm{s} 15 \mathrm{LOX}, 50 \mathrm{mM}$ AAPH, $10 \mu \mathrm{M}$ hemin, or $50 \mathrm{mM}$ AAPH $+10 \mu \mathrm{M}$ hemin. Data are mean $\pm \mathrm{s} . d$. and experiments were performed in triplicate. 


\section{Supporting information Figure S5}
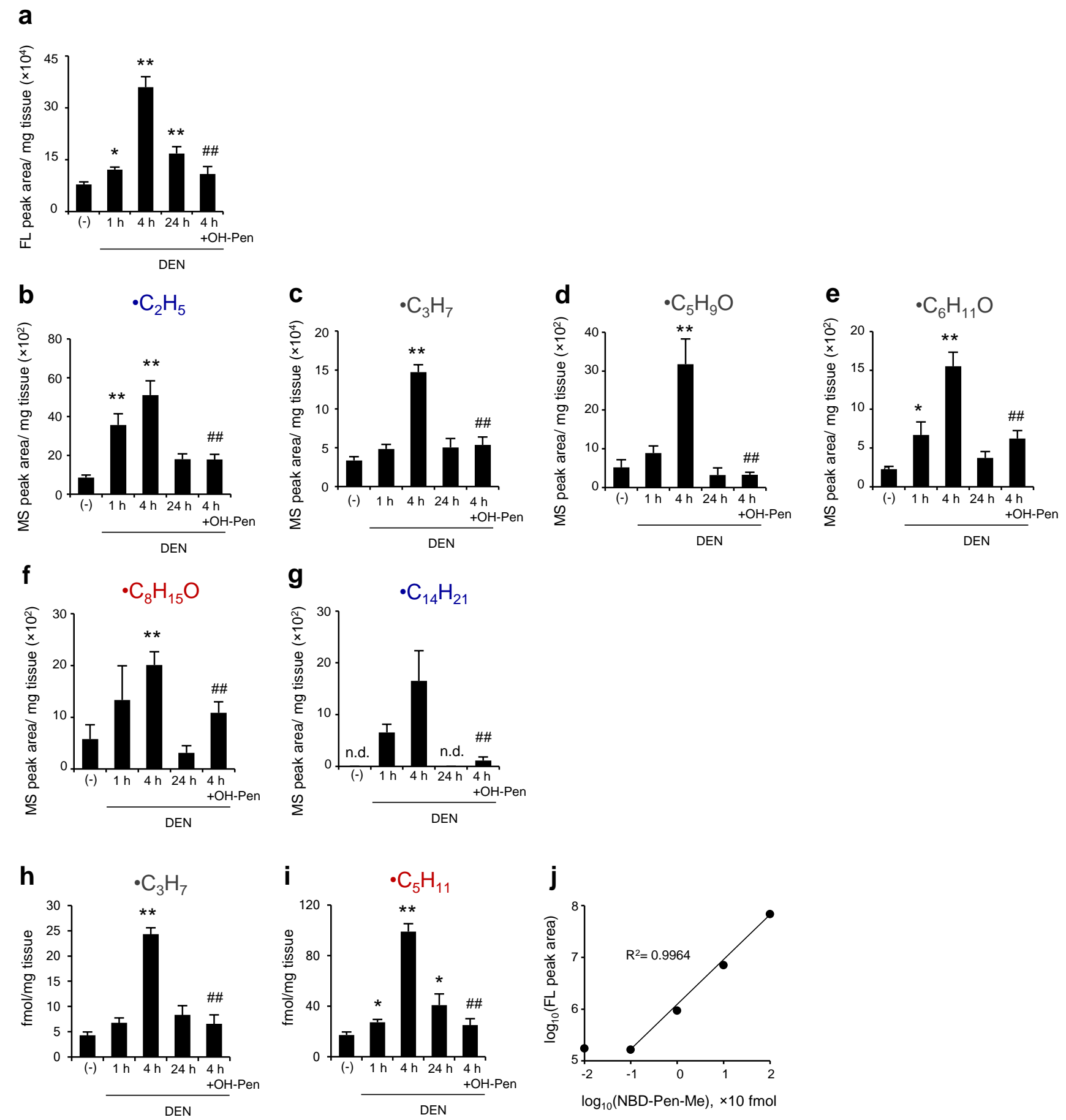

Figure S5 | Change in endogenous lipid-derived radicals in the livers of diethylnitrosamine (DEN) treated mice. (a) Total areas of observed fluorescent peaks of NBD-Pen adducts in liver extracts sampled 1, 4, and $24 \mathrm{~h}$ after DEN injection (100 $\mathrm{mg} / \mathrm{kg}$ ). Mice were administered the lipid radical scavenger, OH-Pen, 15 min before NBD-Pen injection. (b-g) Semiquantified lipid-derived radical products calculated from the EIC peak areas in liver extracts sampled 1, 4, and $24 \mathrm{~h}$ after DEN injection. (h, i) Quantitative analysis of NBD-Pen- $\mathrm{C}_{3} \mathrm{H}_{7}$ and NBD-Pen- $\mathrm{C}_{5} \mathrm{H}_{11}$ in lipid extracts, calculated from each fluorescent peak area. (j) Methoxyamine form of NBD-Pen (NBD-Pen-NOMe) was used as the fluorescence standard compound. Data are mean \pm s.d. and experiments were performed six times. $* P<0.05$, $* * P<0.005$, as compared with the vehicle group. ${ }^{\#} P<0.05,{ }^{\# \#} P<0.005$, as compared with the DEN-treated group after $4 \mathrm{~h}$. 


\section{Supporting information Figure S6}

a
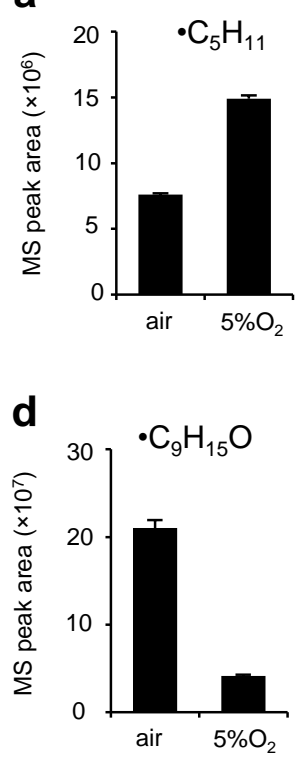

b

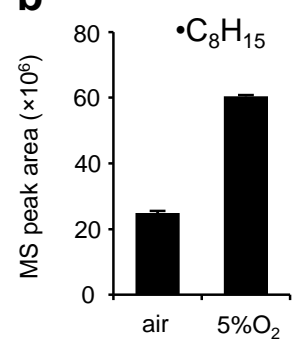

e

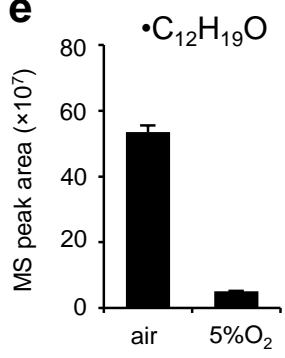

C
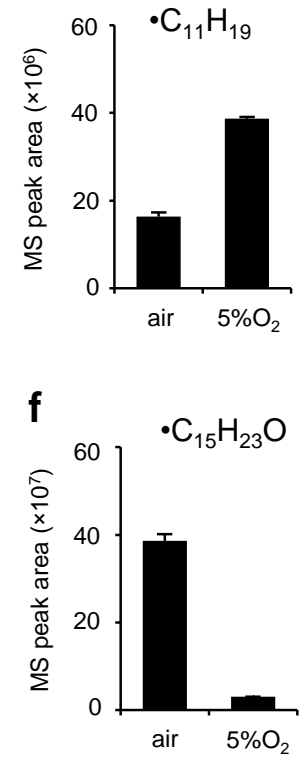

Figure S6 | Semi-quantified lipid-derived radical products stimulated by AAPH in air and $5 \% \mathrm{O}_{2}$-saturated conditions. AA $(500 \mu \mathrm{M})$ was the substrate and AAPH $(50 \mathrm{mM})$ was the stimulator. The $5 \% \mathrm{O}_{2}$-saturated reaction solution was degassed by a vacuum dryer and purged using mixed gas $\left(95 \% \mathrm{~N}_{2}\right.$ and $\left.5 \% \mathrm{O}_{2}\right)$ for 10 min, two times. (a) $\bullet \mathrm{C}_{5} \mathrm{H}_{11}$, (b) $\bullet \mathrm{C}_{8} \mathrm{H}_{15},(\mathbf{c}) \cdot \mathrm{C}_{11} \mathrm{H}_{19}$, (d) $\bullet \mathrm{C}_{9} \mathrm{H}_{15} \mathrm{O}$, (e) $\bullet \mathrm{C}_{12} \mathrm{H}_{19} \mathrm{O}$, and (f) $\bullet \mathrm{C}_{15} \mathrm{H}_{23} \mathrm{O}$. Data are mean \pm s.d. and experiments were performed in triplicate. 


\section{Supporting information Scheme S1}

a

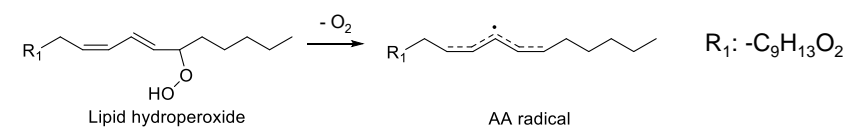

b

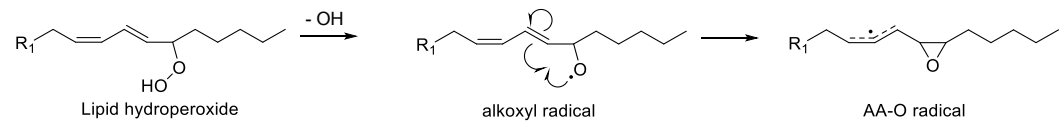

C

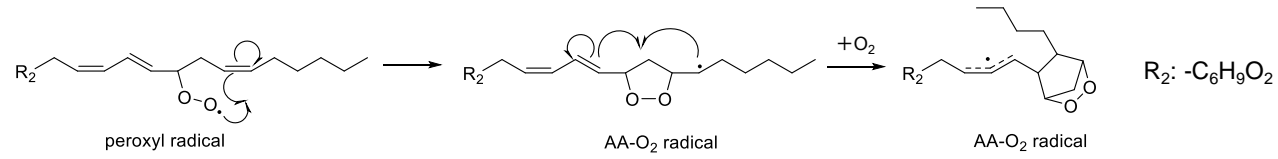

Scheme S1. | Proposed generation mechanism for AA-derived, full-length radicals. (a) AA radical, (b) AA-O radical, and (c) $\mathrm{AA}-\mathrm{O}_{2}$ radicals. 
Table S1 | Change in total fluorescence peak area of 4-(4-nitrobenzo[1,2,5]oxadiazol-7-ylamino)-6pentyl-2,2,6-trimethylpiperidine-1-oxyl (NBD-Pen) -lipid radical adducts under various storage conditions.

\begin{tabular}{cccc}
\hline \multicolumn{4}{c}{ Fluorescence intensity of $>$ NOR, $\%$} \\
\\
\hline at $25^{\circ} \mathrm{C}$ & $84.4 \pm 2.1$ & 1 week & 1 month \\
at $4{ }^{\circ} \mathrm{C}$ & $99.2 \pm 0.1$ & $72.4 \pm 2.0$ & $44.4 \pm 1.2$ \\
at $-30^{\circ} \mathrm{C}$ & $99.5 \pm 1.1$ & $96.7 \pm 0.5$ & $93.1 \pm 2.1$ \\
\hline
\end{tabular}

The reaction mixture was extracted by the Bligh and Mayer method after addition of $10 \mu \mathrm{g} / \mathrm{mL}$ s $15 \mathrm{LOX}$ to a solution of AA and NBD-Pen. The organic solvent was removed by a vacuum dryer, and the residue was dissolved in acetonitrile. The extraction solution was stored at 25,4 , and $-30^{\circ} \mathrm{C}$ from $1 \mathrm{~d}$ to 1 month before LC-FL/MS measurement. We calculated the $\%$ change of the fluorescence peak area of NBD-Pen adducts under several storage conditions based on the initial peak area shown in Figure 1c as 100\%. The conditions were the same as described in Figure S1. Data are mean \pm s. d. and experiments were performed in triplicate. 
Table S2 I Lists of NBD-Pen-PUFA (AA, LA, ALA, DHA, and EPA) derived radical adducts generated in s15LOX, AAPH, or AAPH/Hemin induced peroxidation (yellow; unreported radical before our experiments).

\begin{tabular}{|c|c|c|c|c|c|c|c|c|c|c|c|c|c|}
\hline ID & Formula & Monoisotopic mass & 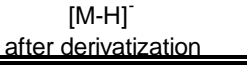 & $t_{p}, \min$ & Observed precursor ion, $m / 2$ & $\begin{array}{l}\text { Mass accuracy, } \\
\text { ppm }\end{array}$ & $\begin{array}{l}\text { Observed fragment } \\
\text { ion, } m z\end{array}$ & $\begin{array}{l}\text { Observed neutral } \\
\text { loss, Da }\end{array}$ & $\begin{array}{l}\text { Massa sccuracy } \\
\text { for neutral loss, pom }\end{array}$ & $\begin{array}{l}\text { MS peak area (n=3) } \\
\text { s15LOX, Average }\end{array}$ & & & \\
\hline & $\overline{C^{\circ} \mathrm{C}_{20} \mathrm{H}_{31} \mathrm{O}_{2}}$ & & & $\begin{array}{l}30-45 \text { (multi) } \\
\end{array}$ & $\begin{array}{lll}692.4400 \\
\end{array}$ & $\begin{array}{ll}1.86 \\
\end{array}$ & $\begin{array}{l}389.2075 \\
\end{array}$ & $\begin{array}{l}303.2325 \\
\end{array}$ & 0.48 & & (12177255 & & \\
\hline 2 & ${ }^{-} \mathrm{C}_{20} \mathrm{H}_{29} \mathrm{O}_{3}$ ( & & & & & & & & -2.90 & & & & \\
\hline 3 & 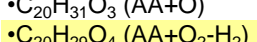 & $\begin{array}{l}319.92733 \\
322206\end{array}$ & 70 & $\begin{array}{l}25-35 \text { (multit) } \\
{ }_{25}\end{array}$ & & $\begin{array}{l}0.25 \\
0.27\end{array}$ & & $\begin{array}{l}319 \\
322\end{array}$ & $\begin{array}{l}-1.32 \\
-1.32\end{array}$ & $\begin{array}{l}250022414640 \\
\text { 2581094 }\end{array}$ & & & 25773120 \\
\hline $\begin{array}{l}4 \\
5\end{array}$ & 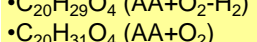 & 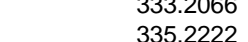 & $\begin{array}{l}72.24744 \\
724291\end{array}$ & 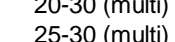 & 17 & $\begin{array}{l}2.47 \\
0.22\end{array}$ & 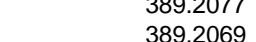 & $\begin{array}{l}335218 \\
3352218\end{array}$ & 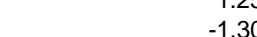 & $\begin{array}{l}1994649016 \\
19016\end{array}$ & 22739285 & $\begin{array}{l}17599364 \\
3657710\end{array}$ & $\begin{array}{l}3355000 \\
150918 \\
150918\end{array}$ \\
\hline 6 & ${ }_{-0}^{-2}$ & $\begin{array}{l}349.2015 \\
34015\end{array}$ & 738.4084 & 20-30 (multi) & 8.4080 & 0.29 & $\begin{array}{l}389.2074 \\
38074\end{array}$ & $\begin{array}{l}\text { S49.2007 } \\
340207\end{array}$ & $-2.43^{2}$ & $\begin{array}{l}3460988 \\
14608\end{array}$ & 195743 & ${ }_{34471340}$ & $\begin{array}{l}536741 \\
540518\end{array}$ \\
\hline 7 & $-{ }^{-} \mathrm{C}_{20} \mathrm{H}_{31} \mathrm{O}_{5}\left(\mathrm{AA}+\mathrm{O}_{3}\right)$ & 351.2171 & 740.4240 & 20-30 (multi) & 72 & 1.17 & 389.2078 & 351.2165 & $-170 \quad-\quad>$ & 49209263 & 3680287 & & 2356323 \\
\hline & ${ }^{-} \mathrm{C}_{20} \mathrm{H}_{31} \mathrm{O}_{6}\left(\mathrm{AA}+\mathrm{O}_{4}\right)$ & 367.2121 & 756.4189 & 20-30 (multi) & 756.4205 & 2.86 & 389.2079 & 367.2126 & $166>$ & 26941875 & 1733538 & 3776512 & 10431 \\
\hline
\end{tabular}

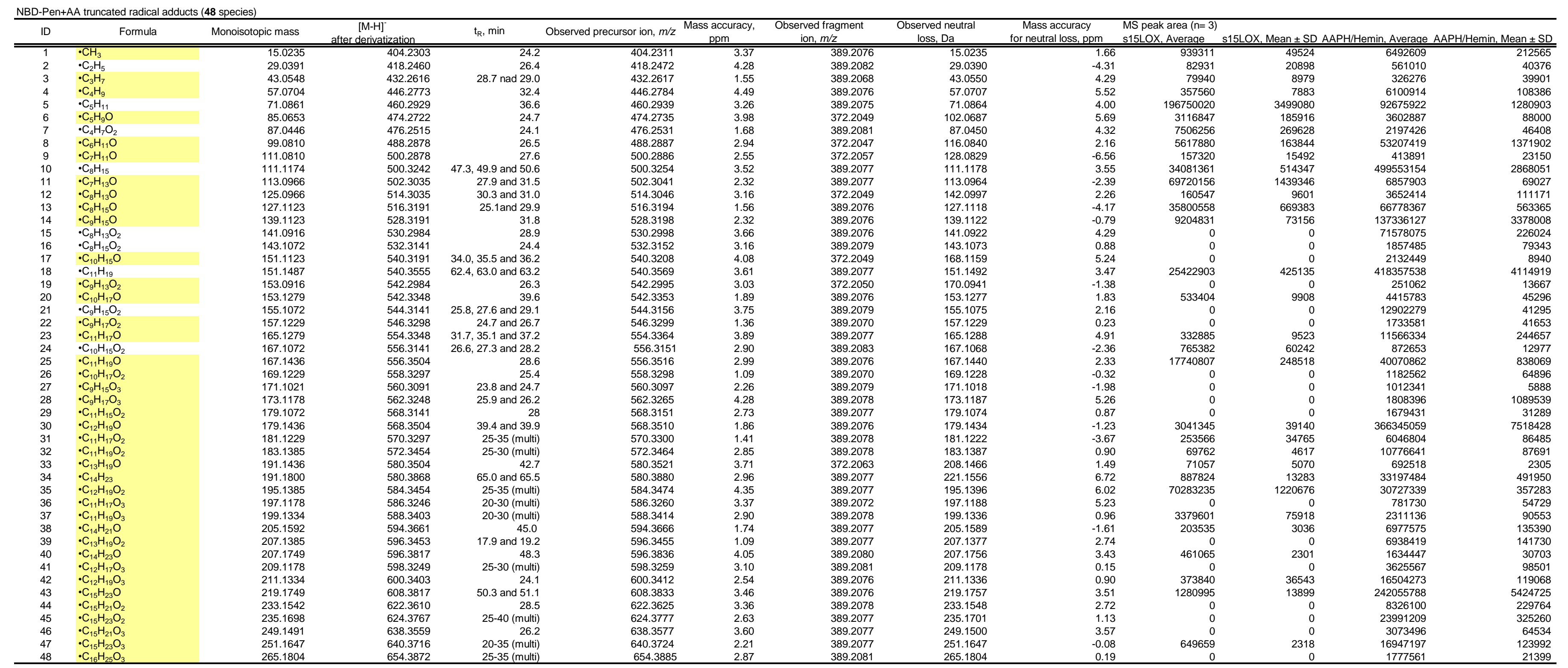

NBD-Pen+LA full-length radical adducts (6 species)

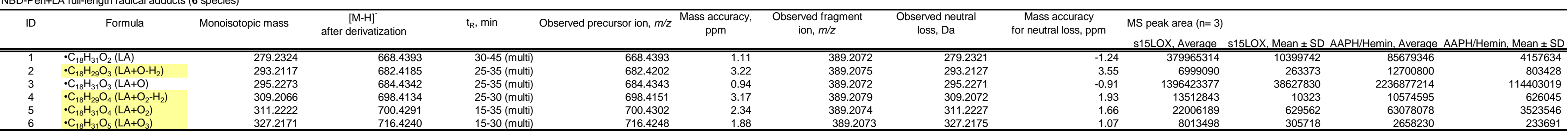

NBD-Pen+LA truncated radical adducts (20 species)

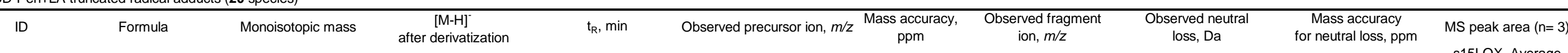

S15LOX.Average S15LOX, Mean \pm SD AAPHHemin, Average AAPHHHemin. Mean \pm SD 


\begin{tabular}{|c|c|c|c|c|c|c|c|c|c|c|c|c|c|}
\hline 1 & $\cdot{ }^{\cdot \mathrm{CH}_{3}}$ & 15.0235 & 404.2303 & 24.2 & 404.2311 & 3.37 & 389.2076 & 15.0235 & 3.66 & 9883 & 1020 & 865324 & 40743 \\
\hline 2 & $\cdot \mathrm{C}_{2} \mathrm{H}_{5}$ & 29.0391 & 418.2460 & 26.5 & 418.2465 & 2.54 & 3899.2074 & 29.0391 & -2.24 & 11260 & 10241 & 426968 & 25559 \\
\hline 4 & $\begin{array}{ll}{ }^{\circ} \mathrm{C}_{3} \mathrm{H}_{7} \mathrm{H}_{7} \\
\mathrm{C}_{4} \mathrm{H}_{9}\end{array}$ & $\begin{array}{l}43.0548 \\
57.0704\end{array}$ & $\begin{array}{l}\begin{array}{l}432.2616 \\
446.2773\end{array} \\
\text {. }\end{array}$ & $\begin{array}{l}28.5 \text { and } 28.9 \\
32.5\end{array}$ & $\begin{array}{l}\begin{array}{l}432.2626 \\
446.2776\end{array} \\
\text { (n) }\end{array}$ & $\begin{array}{l}3.59 \\
2.74\end{array}$ & $\begin{array}{l}389.2082 \\
3392.2073 \\
\end{array}$ & $\begin{array}{l}43.5450 \\
57.0706\end{array}$ & $\begin{array}{c}-7.09 \\
3.41\end{array}$ & $\begin{array}{r}0 \\
551862\end{array}$ & $\begin{array}{r}0 \\
13972\end{array}$ & $\begin{array}{r}168295 \\
924071\end{array}$ & $\begin{array}{r}58539 \\
385780\end{array}$ \\
\hline $\begin{array}{l}4 \\
5\end{array}$ & 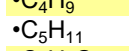 & 71.0861 & $\begin{array}{l}460.2929 \\
4629\end{array}$ & $\begin{array}{l}32.5 \\
36.4\end{array}$ & $\begin{array}{l}440.0710 \\
460.2935\end{array}$ & 2.54 & $\begin{array}{l}3899.2073 \\
3073\end{array}$ & 71.0863 & $\begin{array}{l}3.41 \\
2.74\end{array}$ & $\begin{array}{r}5518622 \\
531107287\end{array}$ & $\begin{array}{r}139 / 2 \\
20126004\end{array}$ & $\begin{array}{l}92444711 \\
64139269\end{array}$ & $\begin{array}{r}368780 \\
1684616\end{array}$ \\
\hline 6 & 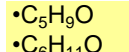 & $\begin{array}{l}85.0653 \\
990810\end{array}$ & $\begin{array}{l}\begin{array}{l}474.2722 \\
4882878\end{array} \\
4\end{array}$ & 24.8 & $\begin{array}{l}474.2734 \\
1808295\end{array}$ & 3.72 & $\begin{array}{l}372.2048 \\
2720206\end{array}$ & $\begin{array}{l}102.0686 \\
111.0892\end{array}$ & 5.10 & $\begin{array}{l}3172082 \\
3120501\end{array}$ & 66333 & $\begin{array}{r}3723113 \\
{ }_{12305726}\end{array}$ & $\begin{array}{r}149500 \\
2802309\end{array}$ \\
\hline & 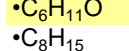 & $\begin{array}{r}99.0810 \\
111.1744\end{array}$ & $\begin{array}{l}\begin{array}{l}488.2878 \\
50.3242\end{array} \\
\end{array}$ & $\begin{array}{l}47.7,50.5 \text { and } 51.6 \\
51.0\end{array}$ & $\begin{array}{l}\begin{array}{l}488.88885 \\
5003250\end{array} \\
\end{array}$ & $\begin{array}{l}2.49 \\
2.60\end{array}$ & $\begin{array}{l}3 / 2.2046 \\
389.2073\end{array}$ & 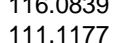 & $\begin{array}{l}1.64 \\
2.74\end{array}$ & $\begin{array}{r}13835044 \\
695248\end{array}$ & $\begin{array}{l}90699 \\
11772\end{array}$ & $\begin{array}{l}43395 / 36 \\
31225287\end{array}$ & $\begin{array}{l}{ }_{2893396} 89347 \\
114317\end{array}$ \\
\hline $\begin{array}{l}8 \\
9\end{array}$ & 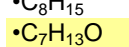 & $\begin{array}{l}111.1174 \\
113.0966\end{array}$ & $\begin{array}{l}500.32424 \\
502355\end{array}$ & $\begin{array}{l}47 . I, 50.5 \text { and } 31.0 \\
27.8\end{array}$ & $\begin{array}{l}502.32041 \\
502.320\end{array}$ & $\begin{array}{l}2.30 \\
2.32\end{array}$ & $\begin{array}{l}3899.2074 \\
38974\end{array}$ & 113.0967 & $\begin{array}{l}2.14 \\
0.62\end{array}$ & $\begin{array}{l}5287863 \\
52833\end{array}$ & 143662 & $\begin{array}{r}\quad 1827479 \\
182796\end{array}$ & 65523 \\
\hline 10 & $\cdot{ }^{-} \mathrm{C}_{8} \mathrm{H}_{13} \mathrm{O}$ & 125.0966 & 514.3035 & 30.4 and 31.1 & 514.3044 & 2.79 & 372.2056 & 142.0988 & -3.94 & 0 & 0 & 1419273 & 109193 \\
\hline 11 & $\cdot \mathrm{C}_{8} \mathrm{H}_{15} \mathrm{O}$ & 127.1123 & 516.3191 & 25.2 and 30.0 & 516.3194 & 1.56 & 389.2074 & 127.1120 & -2.52 & 1541697 & 9815 & 6035483 & 423018 \\
\hline $\begin{array}{l}12 \\
13\end{array}$ & 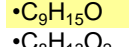 & $\begin{array}{l}139.1123 \\
1410916\end{array}$ & $\begin{array}{l}528.3191 \\
5320984\end{array}$ & $\begin{array}{l}31.9 \\
200\end{array}$ & $\begin{array}{l}528.3195 \\
5320991\end{array}$ & $\begin{array}{l}1.75 \\
228\end{array}$ & $\begin{array}{l}389.2073 \\
3892075\end{array}$ & $\begin{array}{l}139.1122 \\
1410916\end{array}$ & $\begin{array}{l}-0.36 \\
0.39\end{array}$ & 7064571 & 146031 & $\begin{array}{l}207787203 \\
11962199\end{array}$ & 11129994 \\
\hline $\begin{array}{l}13 \\
14\end{array}$ & 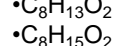 & $\begin{array}{l}\begin{array}{l}144.0916 \\
143.1072\end{array} \\
\end{array}$ & $\begin{array}{l}\begin{array}{l}530.02984 \\
532.3141\end{array} \\
\end{array}$ & 294.5 & $\begin{array}{l}\begin{array}{l}530.02991 \\
532.3151\end{array} \\
\end{array}$ & $\begin{array}{l}2.28 \\
3.03\end{array}$ & $\begin{array}{l}389.2075 \\
399.2075\end{array}$ & $\begin{array}{l}\begin{array}{l}144.0916 \\
143.1076\end{array} \\
\end{array}$ & $\begin{array}{l}0.39 \\
2.76\end{array}$ & $\begin{array}{l}0 \\
0\end{array}$ & $\begin{array}{l}0 \\
0 \\
0\end{array}$ & $\begin{array}{l}11962197 \\
457046\end{array}$ & $\begin{array}{l}698723 \\
57830\end{array}$ \\
\hline 15 & 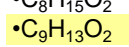 & $\begin{array}{l}\text { 155.0916 } \\
15309\end{array}$ & $\begin{array}{l}542.2984 \\
5428\end{array}$ & 26 & $\begin{array}{l}542.2997 \\
5\end{array}$ & $\begin{array}{l}\text { r.s. } \\
3.47\end{array}$ & 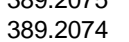 & 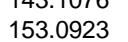 & 5.20 & 0 & 0 & $\begin{array}{l}543690 \\
54369\end{array}$ & $\begin{array}{l}301906 \\
30196\end{array}$ \\
\hline 6 & - ${ }^{-} \mathrm{C}_{9} \mathrm{H}_{15} \mathrm{O}_{2} \mathrm{O}_{2}$ & 155.1072 & 544.3141 & 29.2 & 544.3150 & 2.74 & 389.2072 & 155.1078 & 3.58 & 325592 & 16080 & 13320180 & 585155 \\
\hline & $\cdot{ }^{\cdot \mathrm{C}_{9} \mathrm{H}_{1} 7 \mathrm{O}_{2}}$ & 157.1229 & 546.32297 & 26.9 & 546.3298 & 0.00 & 389.2074 & 157.1224 & -3.02 & 0 & 0 & 690476 & 34034 \\
\hline 8 & $\begin{array}{l}{ }^{-} \mathrm{C}_{9} \mathrm{H}_{15} \mathrm{O}_{3} \\
. \mathrm{C}_{0} \mathrm{H}_{1} \mathrm{O}_{2}\end{array}$ & $\begin{array}{l}177.1021 \\
171.117\end{array}$ & $\begin{array}{l}560.309090 \\
56034\end{array}$ & $\begin{array}{l}23.9 \text { and } 24.8 \\
24.8\end{array}$ & $\begin{array}{l}560.309090 \\
5603250\end{array}$ & $\begin{array}{l}0.00 \\
0.00\end{array}$ & 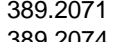 & $\begin{array}{l}177.10199 \\
1.11192\end{array}$ & $\begin{array}{l}-1.146 \\
-1.46\end{array}$ & $\begin{array}{l}0 \\
0\end{array}$ & $\begin{array}{l}0 \\
0\end{array}$ & $\begin{array}{l}380496 \\
55047\end{array}$ & $\begin{array}{l}47627 \\
45669\end{array}$ \\
\hline 20 & 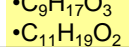 & $\begin{array}{l}183.1785 \\
183.118\end{array}$ & $\begin{array}{l}502.22346 \\
572.3454\end{array}$ & $\begin{array}{l}26.0 \text { and (26.2.2. } \\
15-20 \text { (mult) }\end{array}$ & $\begin{array}{l}572.3466 \\
57466\end{array}$ & $\begin{array}{l}3.20 \\
3.16\end{array}$ & $\begin{array}{l}\text { 385.2074 } \\
\text { 3. }\end{array}$ & $\begin{array}{l}183.1393 \\
183.1393\end{array}$ & $\begin{array}{l}4.05 \\
4.07\end{array}$ & $\begin{array}{l}0 \\
0\end{array}$ & $\begin{array}{l}0 \\
0\end{array}$ & $\begin{array}{l}4597892 \\
45972\end{array}$ & $\begin{array}{l}2535197 \\
2\end{array}$ \\
\hline
\end{tabular}

NBD-Pen+ALA full-ength radical adducts 6 species

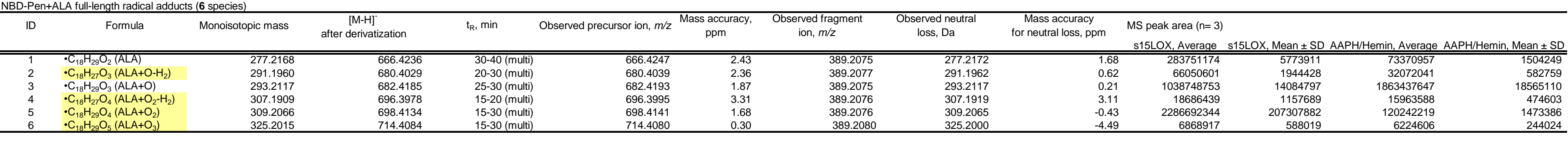

NBD-Pen+ALA truncated radical adducts (22 species)

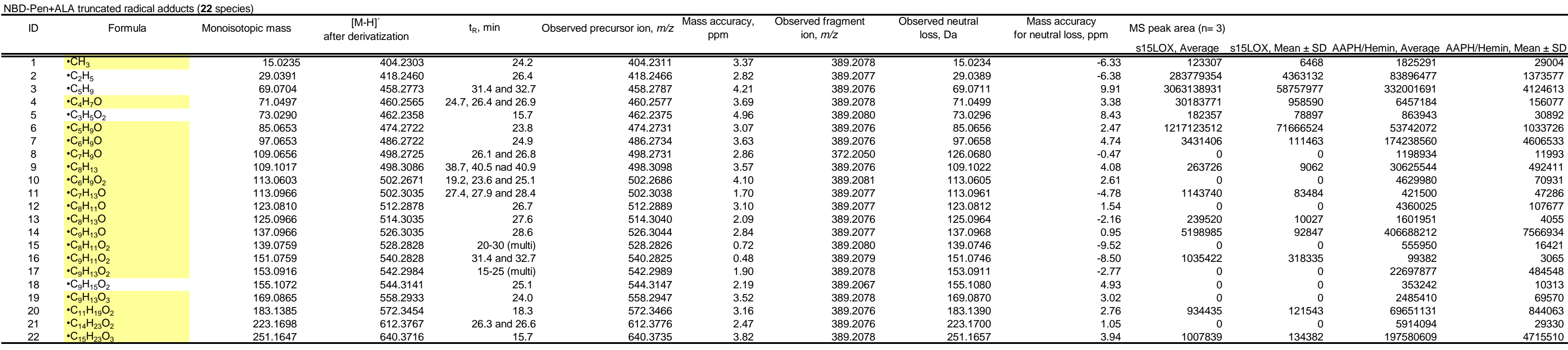

NBD-Pen+DHA full-ength radical adducts (8 species)

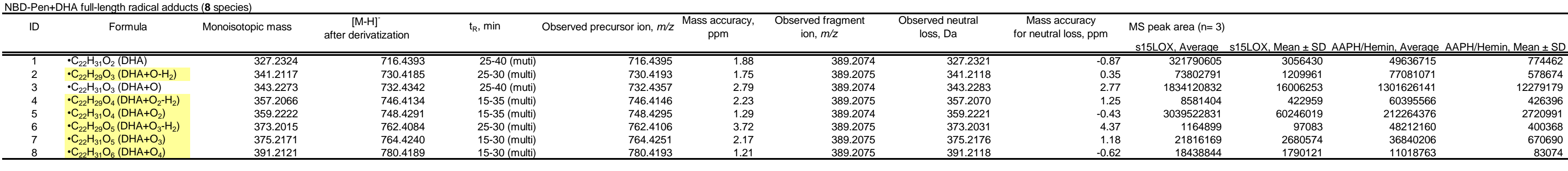

NBD-Pen+DHA truncated radical adducts (64 species

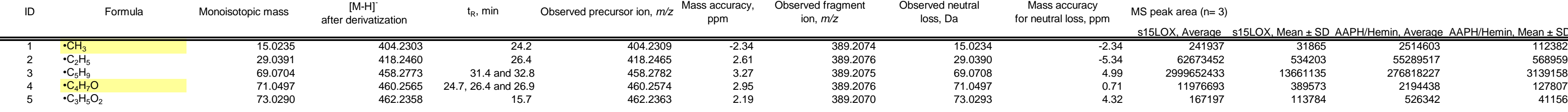




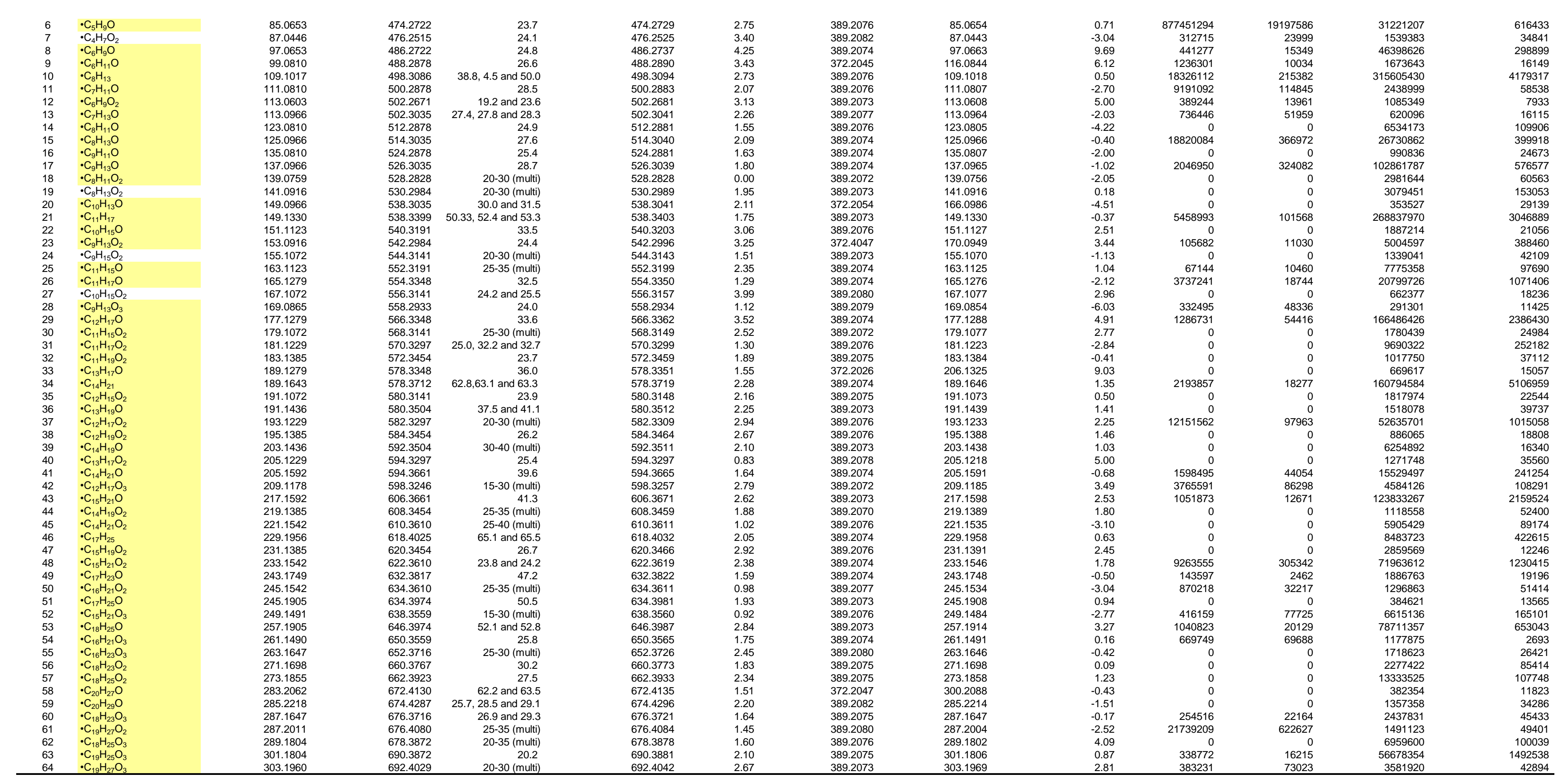

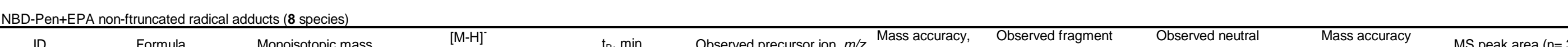

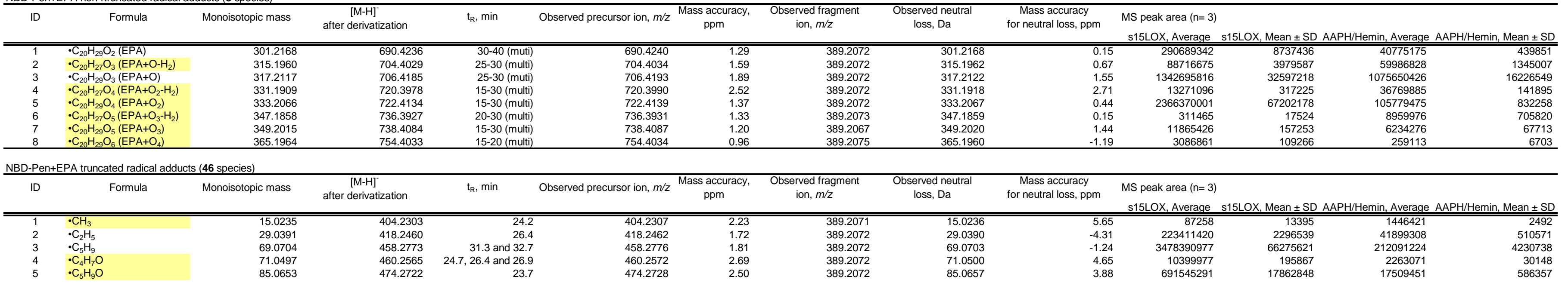




\begin{tabular}{|c|c|c|c|c|c|c|c|c|c|c|c|c|c|}
\hline 6 & $\cdot \mathrm{C}_{4} \mathrm{H}_{7} \mathrm{O}_{2}$ & 87.0446 & 476.2515 & 24.1 & 476.2521 & 2.59 & 389.2070 & 87.0451 & 5.69 & 87622 & 11928 & 316165 & 4903 \\
\hline 7 & $\cdot \mathrm{C}_{6} \mathrm{H}_{9} \mathrm{O}$ & $\begin{array}{l}97.0653 \\
000010\end{array}$ & $\begin{array}{l}486.2722 \\
1802809\end{array}$ & 24.8 & $\begin{array}{l}486.2730 \\
102008\end{array}$ & $\begin{array}{l}2.87 \\
2.10\end{array}$ & $\begin{array}{l}389.2071 \\
{ }_{3}^{2} 20018\end{array}$ & $\begin{array}{r}97.0659 \\
.11050\end{array}$ & 5.98 & $\begin{array}{r}934435 \\
601015\end{array}$ & $\begin{array}{r}38925 \\
252170\end{array}$ & 82338158 & 1046565 \\
\hline 8 & $\begin{array}{l}\cdot{ }^{\circ} \mathrm{CH}_{11} \mathrm{O} \\
\cdot \mathrm{C}_{-} \mathrm{H}_{0} \mathrm{O}\end{array}$ & $\begin{array}{r}99.0810 \\
100653\end{array}$ & $\begin{array}{l}488.2878 \\
4927272\end{array}$ & 26.6 & 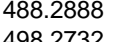 & $\begin{array}{l}3.19 \\
304\end{array}$ & $\begin{array}{l}372.2048 \\
372044\end{array}$ & $\begin{array}{l}116.08840 \\
10.098\end{array}$ & $\begin{array}{l}2.76 \\
{ }_{516}\end{array}$ & 4601215 & 253470 & $\begin{array}{l}1460148 \\
545478\end{array}$ & $\begin{array}{l}41346 \\
27000\end{array}$ \\
\hline $\begin{array}{l}9 \\
10\end{array}$ & $\begin{array}{l}{ }^{\circ}{ }^{-} \mathrm{C}_{8} \mathrm{H}_{9} \mathrm{O} \\
\mathrm{H}_{13}\end{array}$ & $\begin{array}{l}\begin{array}{l}109.96533 \\
109.1017\end{array} \\
\end{array}$ & $\begin{array}{l}498.8 / 2226 \\
4983.3086\end{array}$ & $38.8,40.5$ and 40.9 & $\begin{array}{l}4988.27323 \\
49980\end{array}$ & $\begin{array}{l}3.04 \\
1.93\end{array}$ & $\begin{array}{l}3 / 2.2044 \\
389.2076\end{array}$ & $\begin{array}{l}\begin{array}{l}122.0688 \\
109.1014\end{array} \\
1\end{array}$ & $\begin{array}{r}5.16 \\
-2.62\end{array}$ & $\begin{array}{l}0 \\
7531315\end{array}$ & $10315^{\circ}$ & $\begin{array}{l}5158448.8 \\
2158194\end{array}$ & $\begin{array}{l}20000 \\
90433\end{array}$ \\
\hline 11 & 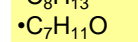 & $\begin{array}{l}111.0810 \\
110\end{array}$ & $\begin{array}{l}500.2878 \\
5078\end{array}$ & $\begin{array}{ll}0.0,0,0.0 .5 \\
28.5\end{array}$ & $\begin{array}{l}4000.2882 \\
508.309\end{array}$ & 1.83 & $\begin{array}{l}389.2076 \\
389.2073\end{array}$ & $\begin{array}{l}10.91044 \\
111.0809\end{array}$ & $\begin{array}{l}-1.08 \\
-1.08 \\
\end{array}$ & $\begin{array}{l}9319509 \\
931950\end{array}$ & 2211188 & $\begin{array}{c}2.801794 \\
147034\end{array}$ & $\begin{array}{l}904313 \\
9169\end{array}$ \\
\hline 12 & $\cdot{ }_{\mathrm{C}_{6}} \mathrm{H}_{9} \mathrm{O}_{2}$ & 113.0603 & 502.2671 & 19.1 and 23.6 & 502.2677 & 2.21 & 389.2072 & 113.0605 & 1.82 & 579843 & 15372 & 2352335 & 29905 \\
\hline 13 & $\cdot{ }^{-C_{8} H_{11} \mathrm{O}}$ & $\begin{array}{l}123.0810 \\
1250965\end{array}$ & $\begin{array}{l}512.2878 \\
513025\end{array}$ & 24.9 & $\begin{array}{l}512.2876 \\
513013\end{array}$ & 0.60 & $\begin{array}{l}389.2072 \\
3802072\end{array}$ & $\begin{array}{l}123.0804 \\
1250070\end{array}$ & -4.47 & 0 & 0 & 4701986 & 163862 \\
\hline $\begin{array}{l}14 \\
15\end{array}$ & $\begin{array}{l}{ }^{\circ}{ }_{8}^{8} \mathrm{H}_{13} \mathrm{O} \\
\mathrm{C}_{9} \mathrm{H}_{13} \mathrm{O}\end{array}$ & $\begin{array}{l}\begin{array}{l}25.50966 \\
13.0966\end{array} \\
\end{array}$ & $\begin{array}{l}514.3035 \\
52633035\end{array}$ & $\begin{array}{l}27.6 \\
28.6\end{array}$ & $\begin{array}{l}524.3043 \\
526.3036\end{array}$ & $\begin{array}{l}2.56 \\
1.34\end{array}$ & $\begin{array}{l}389.2072 \\
38292072\end{array}$ & $\begin{array}{l}\begin{array}{l}125.0970 \\
137.0965\end{array} \\
\end{array}$ & $\begin{array}{r}3.28 \\
-1.02 \\
\end{array}$ & $\begin{array}{l}2026694 \\
3048816\end{array}$ & $\begin{array}{l}109187 \\
66098\end{array}$ & $\begin{array}{r}10596654 \\
174330889\end{array}$ & $\begin{array}{r}344318 \\
109576\end{array}$ \\
\hline 16 & 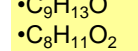 & $\begin{array}{l}137.9066 \\
139.0759\end{array}$ & $\begin{array}{l}52.63035 \\
5282828\end{array}$ & $\begin{array}{l}28.6 \\
20-30 \text { (multi) }\end{array}$ & 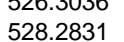 & $\begin{array}{l}1.34 \\
1.65\end{array}$ & 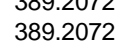 & $\begin{array}{l}13.0758 \\
13.0758\end{array}$ & 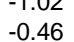 & $\begin{array}{l}378000 \\
878030\end{array}$ & $\begin{array}{l}{ }_{182025} \\
18225\end{array}$ & $\begin{array}{l}1773308899 \\
28357\end{array}$ & $\begin{array}{l}1096676 \\
7740\end{array}$ \\
\hline 17 & $\cdot{ }^{-6} \mathrm{C}_{10} \mathrm{H}_{13} \mathrm{O}_{2}$ & 149.0966 & 538.3035 & 20=00 (IIt) 30 & $\begin{array}{l}528.3036 \\
50.0031\end{array}$ & $\begin{array}{l}1.05 \\
1.20\end{array}$ & $\begin{array}{l}372.2050 \\
372.050\end{array}$ & $\begin{array}{l}156.0986 \\
16960\end{array}$ & $\begin{array}{l}-4.40 \\
-4.51\end{array}$ & 0 & $\begin{array}{r}10<20 \\
0\end{array}$ & 645394 & 8162 \\
\hline 18 & $\cdot \mathrm{C}_{11} \mathrm{H}_{17}$ & 149.1330 & 538.3399 & $50.3,52.3$ and 53.2 & 538.3405 & 2.21 & 389.2072 & 149.1334 & 2.24 & 1859988 & 36878 & 152979474 & 1641719 \\
\hline 19 & $\cdot \mathrm{C}_{10} \mathrm{H}_{15} \mathrm{O}$ & $\begin{array}{l}151.1123 \\
152016\end{array}$ & $\begin{array}{l}540.3191 \\
502009\end{array}$ & & 540.3201 & 2.84 & $\begin{array}{l}389.2074 \\
272014\end{array}$ & 151.11128 & 3.11 & & 0 & 1151545 & 12144 \\
\hline $\begin{array}{l}20 \\
21\end{array}$ & 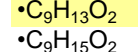 & $\begin{array}{l}\begin{array}{l}153.0916 \\
155.1027\end{array} \\
1\end{array}$ & $\begin{array}{l}542.2984 \\
544.3141\end{array}$ & $\begin{aligned} 24.4 \\
20-30 \text { (multi) }\end{aligned}$ & $\begin{array}{l}542.2992 \\
544.3137\end{array}$ & $\begin{array}{l}2.46 \\
0.28\end{array}$ & $\begin{array}{l}\begin{array}{l}372.2044 \\
389.2072\end{array} \\
3807\end{array}$ & $\begin{array}{l}170.0948 \\
1551064\end{array}$ & $\begin{array}{r}2.92 \\
-512\end{array}$ & $\begin{array}{l}0 \\
0 \\
0\end{array}$ & $\begin{array}{l}0 \\
0\end{array}$ & $\begin{array}{r}4276251 \\
635955\end{array}$ & $\begin{array}{r}259430 \\
65090\end{array}$ \\
\hline 22 & $\begin{array}{l}\bullet_{9}^{-}{ }^{9} \mathrm{H}_{15} \mathrm{O}_{2} \\
\mathrm{C}_{11} \mathrm{H}_{15}\end{array}$ & $\begin{array}{l}\begin{array}{l}155.1027 \\
163.1123\end{array} \\
\end{array}$ & $\begin{array}{l}542.3141 \\
552.3191\end{array}$ & $\begin{array}{l}20-30-30 \text { (multit) } \\
25 \text { (muti) }\end{array}$ & $\begin{array}{l}542.313137 \\
552.3193\end{array}$ & $\begin{array}{l}0.28 \\
1.35\end{array}$ & $\begin{array}{l}389.2012 \\
389.2069\end{array}$ & 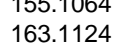 & 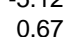 & 84411 & $\begin{aligned} 0 \\
4641\end{aligned}$ & $\begin{array}{l}5350535 \\
53312\end{array}$ & $\begin{array}{l}62899 \\
7872\end{array}$ \\
\hline 23 & $\cdot{ }^{-} C_{11} H_{17} \mathrm{O}$ & 165.1279 & 554.3348 & 32.5 & 554.3353 & 1.96 & 389.2072 & 165.1282 & 1.39 & 1802497 & 18660 & 9887464 & 219042 \\
\hline 24 & $\cdot \mathrm{C}_{10} \mathrm{H}_{15} \mathrm{O}_{2}$ & 167.1072 & 556.3141 & 24.2 and 25.4 & 556.3152 & 3.13 & 389.2071 & 167.1081 & 5.48 & & 0 & 492050 & 11202 \\
\hline 25 & 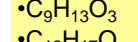 & $\begin{array}{l}16.90865 \\
1777\end{array}$ & $\begin{array}{l}556.2933 \\
5628\end{array}$ & $\begin{array}{l}24.0 \\
237\end{array}$ & $\begin{array}{l}558.2938 \\
56.345\end{array}$ & $\begin{array}{l}1.77 \\
0.50\end{array}$ & $\begin{array}{l}389.2073 \\
382072\end{array}$ & $\begin{array}{l}169.08655 \\
177727\end{array}$ & 0.12 & 0 & $\begin{array}{l}0 \\
50137\end{array}$ & $\begin{array}{r}671942 \\
042016\end{array}$ & $\begin{array}{r}25434 \\
37720914\end{array}$ \\
\hline 年 & 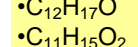 & 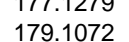 & 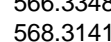 & $\begin{array}{l}25-30 \text { (multiti } \\
2\end{array}$ & 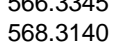 & $\begin{array}{l}0.79 \\
0.79\end{array}$ & 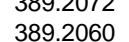 & (17908 & $\begin{array}{l}-3.35 \\
427\end{array}$ & 11163364 & 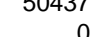 & $\begin{array}{l}2402061350 \\
661328\end{array}$ & $\begin{array}{l}37222981 \\
{ }_{2436}\end{array}$ \\
\hline 28 & 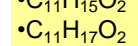 & $\begin{array}{l}181.1229 \\
181.107\end{array}$ & 550.3297 & $25.0,32.2$ and 32.7 & $\begin{array}{l}570.3300 \\
5300\end{array}$ & 1.41 & $\begin{array}{l}389.2073 \\
389.200\end{array}$ & $\begin{array}{l}181.1227 \\
1827\end{array}$ & 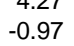 & 0 & 0 & 2979255 & $\begin{array}{l}34300 \\
64658\end{array}$ \\
\hline 29 & $\cdot{ }^{\mathrm{C}_{13} \mathrm{H}_{17} \mathrm{O}^{\circ}}$ & 189.1279 & 578.3348 & 36.0 & 578.3353 & 1.88 & 372.2038 & 206.1315 & 3.98 & & 0 & 263104 & 6135 \\
\hline 30 & $\cdot \mathrm{C}_{14} \mathrm{H}_{21}$ & 189.1643 & 578.3712 & 62.8,63.1 and 63.3 & 578.3719 & 2.18 & 389.2072 & 189.1647 & 1.98 & & 0 & 17254150 & 182668 \\
\hline 列 & 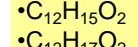 & $\begin{array}{l}191.1072 \\
1931229\end{array}$ & $\begin{array}{l}580.3141 \\
5823297\end{array}$ & $\begin{array}{r}23.9 \\
20-30 . \text { mutit }\end{array}$ & $\begin{array}{l}58.03147 \\
503203\end{array}$ & $\begin{array}{l}2.05 \\
1.89\end{array}$ & $\begin{array}{l}389.2073 \\
38072\end{array}$ & $\begin{array}{l}191.101074 \\
1_{1927}\end{array}$ & ${ }_{1.13}^{1.13}$ & $\begin{array}{r}0 \\
362189\end{array}$ & 7100 & $\begin{array}{l}11449990 \\
174902\end{array}$ & $\begin{array}{l}200499 \\
2009\end{array}$ \\
\hline 33 & 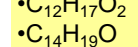 & $\begin{array}{l}\begin{array}{r}193.1229 \\
203.1436\end{array} \\
\end{array}$ & $\begin{array}{l}582.2329 \\
592.3504\end{array}$ & $\begin{array}{l}20-50(\text { IItuIII) } \\
37.4\end{array}$ & 592.3506 & $\begin{array}{l}1.09 \\
1.28\end{array}$ & $\begin{array}{l}389.2072 \\
389.27\end{array}$ & 2013.1434 & $\begin{array}{l}-0.94 \\
-0.11\end{array}$ & $\begin{array}{lll}3021091 \\
0\end{array}$ & $\begin{array}{lll}14 \angle 250 \\
0\end{array}$ & 2014925 & $\begin{array}{l}222284 \\
62155 \\
\end{array}$ \\
\hline 34 & 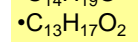 & 205.1229 & 594.3297 & 25.4 & 594.3300 & 1.45 & 389.2075 & 205.1225 & $\begin{array}{l}-.154 \\
-1.58\end{array}$ & 0 & 0 & 607802 & 9111 \\
\hline 35 & $\cdot{ }^{-C_{14} H_{2} O^{\circ}}$ & 205.1592 & 594.3661 & 39.6 & 594.3671 & 2.57 & 389.2074 & 205.1597 & 2.10 & & 0 & 517125 & 16532 \\
\hline 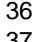 & 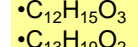 & $\begin{array}{l}207.1021 \\
207.295\end{array}$ & $\begin{array}{l}596.3090 \\
5963454\end{array}$ & $\begin{array}{l}20-40 \text { (muttit) } \\
181 . \text { and } 19\end{array}$ & $\begin{array}{l}596.3098 \\
5963460\end{array}$ & $\begin{array}{l}2.23 \\
201\end{array}$ & $\begin{array}{l}389.2062 \\
38892070\end{array}$ & $\begin{array}{l}207.1035 \\
207.7390\end{array}$ & $\begin{array}{l}6.91 \\
229\end{array}$ & $\begin{array}{l}071767 \\
0\end{array}$ & 0 & $\begin{array}{r}3765709 \\
\quad\end{array}$ & $\begin{array}{r}56831 \\
180000\end{array}$ \\
\hline 列 & 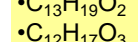 & $\begin{array}{l}2091178 \\
20978\end{array}$ & 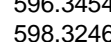 & 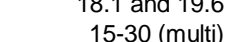 & 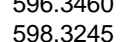 & $\begin{array}{l}2.01 \\
0.65\end{array}$ & 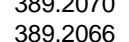 & $\begin{array}{l}20.91190 \\
2009179\end{array}$ & $\begin{array}{l}2.29 \\
0.58\end{array}$ & $2 \pi 160$ & (9) & 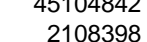 & $\begin{array}{l}1800999 \\
15750\end{array}$ \\
\hline 39 & 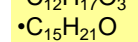 & $\begin{array}{l}217.1592 \\
21592\end{array}$ & $\begin{array}{l}590.32840 \\
606.3661\end{array}$ & 41.3 & $\begin{array}{l}250.3651 \\
606.3651\end{array}$ & -0.72 & $\begin{array}{l}389.2072 \\
38072\end{array}$ & $\begin{array}{l}217.1579 \\
2157\end{array}$ & $\begin{array}{l}-6.17 \\
-617\end{array}$ & 1547614 & 27015 & $\begin{array}{l}175382303 \\
\end{array}$ & $\begin{array}{l}1568742 \\
176872\end{array}$ \\
\hline 40 & $\cdot \mathrm{C}_{1} \mathrm{H}_{25}$ & 229.1956 & 618.4025 & 65.1 and 65.5 & 618.4030 & 1.76 & 389.2072 & 229.1958 & 0.76 & & 0 & 137913 & 24485 \\
\hline 41 & $\cdot \mathrm{C}_{15} \mathrm{C}_{19} \mathrm{O}_{2}$ & 231.1385 & 620.3454 & 26.7 & 620.3461 & 2.13 & 389.2073 & 231.1388 & 1.36 & 0 & 0 & 2666640 & 44406 \\
\hline 42 & $\begin{array}{r}{ }^{-} \mathrm{C}_{15} \mathrm{H}_{21} \mathrm{O}_{2} \\
-\end{array}$ & 233.7542 & 622.3610 & 23.8 and 24.2. & $\begin{array}{l}622.3619 \\
62019\end{array}$ & 2.38 & 389.2073 & 233.1546 & 2.04 & 0 & 0 & $\begin{array}{l}9068358 \\
02370\end{array}$ & $\begin{array}{l}134953 \\
13970\end{array}$ \\
\hline 列 & 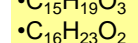 & $\begin{array}{l}24.71 .1354 \\
247.1698\end{array}$ & 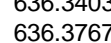 & & 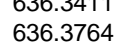 & 046 & $\begin{array}{l}389.2000 \\
389.2072\end{array}$ & $\begin{array}{l}247.7352 \\
247692\end{array}$ & -261 & & 0 & $\begin{array}{l}28534719 \\
3641363\end{array}$ & $\begin{array}{l}1353736 \\
11620\end{array}$ \\
\hline 45 & 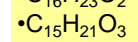 & 249.1491 & 638.3559 & 15-30 (multi) & 638.3563 & 1.39 & $\begin{array}{l}389.2068 \\
3806\end{array}$ & 249.1494 & 1.49 & 198269 & 66501 & 3745812 & 71876 \\
\hline 46 & $\cdot \mathrm{C}_{1} \mathrm{H}_{25} \mathrm{O}_{2}$ & 261.1855 & 650.3923 & $25-30$ (multi) & 650.3930 & 1.92 & 389.2072 & 261.1858 & 1.40 & 29022272 & 1166254 & 592237 & 9093 \\
\hline
\end{tabular}




\section{Methods and Materials}

\section{Reagents}

4-(4-nitrobenzo[1,2,5]oxadiazol-7-ylamino)-6-pentyl-2,2,6-trimethylpiperidine-1-oxyl (NBD-Pen) was synthesised according to a previously reported method ${ }^{1}$. For the lipid radical generation system, arachidonic acid (AA, from porcine liver, $\geq 99 \%$ ), linoleic acid (LA, $\geq 99 \%$ ), $\alpha$-linolenic acid (ALA, $\geq 99 \%$ ), cis-4,7,10,16,19-docosahexaenoic acid (DHA, $\geq 99 \%$ ), cis-5,8,11,14,17-eicosapentaenoic acid (EPA, $\geq 99 \%$ ), and soybean lipoxidase from Glycine max (s15LOX) were purchased from SigmaAldrich (St. Louis, MO), 2,2'-azobis(2-amidinopropane)dihydrochloride (AAPH) from Wako Pure Chemical Industries, Ltd. (Osaka, Japan), and hemin from Tokyo Chemical Industry Co., Ltd. (Tokyo, Japan). For high-performance liquid chromatography (HPLC) analysis, acetonitrile (LCMS grade, $\geq 99.9 \%$ ) was purchased from Nacalai Tesque (Kyoto, Japan), and ammonium acetate (Wako $1^{\text {st }}$ grade) from Wako Pure Chemical Industries, Ltd.

\section{Fluorescent standard curve using methoxyamine form of NBD-Pen (NBD-Pen-NOMe)}

An acetonitrile solution containing given a concentration of NBD-NOMe $(0.1,1,10,100$, and 1000 $\mathrm{nM}$ ) was injected into the HPLC column. The LC conditions were as follows: injection volume, 10 $\mu \mathrm{L}$; autosampler temperature, $4^{\circ} \mathrm{C}$; column, InertSustain $\mathrm{C} 18$ column $(2.1 \times 150 \mathrm{~mm}$, particle size of $3 \mu \mathrm{m}$, GL sciences, Tokyo, Japan); column temperature, $40^{\circ} \mathrm{C}$; mobile phase $5 \mathrm{mM}$ ammonium acetate in $\mathrm{H}_{2} \mathrm{O}$ (A) and $5 \mathrm{mM}$ ammonium acetate in acetonitrile/ $\mathrm{H}_{2} \mathrm{O}(95 / 5)(B)$; flow rate, $0.4 \mathrm{~mL} / \mathrm{min}$; and isocratic elution, $80 \% \mathrm{~B}, 0-15 \mathrm{~min}$. The fluorescence detector parameter settings were the same as given above. The fluorescent peak area of NBD-Pen-NOMe ( $t_{R}: 5.0 \mathrm{~min}$ ) was determined using LabSolutions version 5.80 software (Shimadzu Co.).

\section{AAPH-induced generation system in 5\% $\mathrm{O}_{2}$-saturated or Ar-saturated PBS solution}

A reaction solution containing $500 \mu \mathrm{M} \mathrm{AA}$ (containing $0.5 \%$ ethanol) was degassed by a vacuum dryer and purged using mixed gas $\left(95 \% \mathrm{~N}_{2}\right.$ and $\left.5 \% \mathrm{O}_{2}\right)$ for 10 min twice. After purging, $50 \mathrm{mM}$ AAPH was added to the deoxygenated reaction solution. After $60 \mathrm{~min}, 5.0 \mu \mathrm{M}$ NBD-Pen (containing $0.5 \%$ acetonitrile) was added to the reaction mixture and incubated at room temperature for $15 \mathrm{~min}$. Subsequently, the mixture was extracted by the Bligh and Mayer method ${ }^{2}$.

\section{Statistical analysis}

Statistical analyses were carried out using StatView 5.0 (SAS Institute Inc.), and the data were analysed by the Tukey-Kramer test. The results are expressed as mean \pm standard deviation, and $P<$ 0.05 was considered statistically significant. 


\section{Supplemental reference}

(1) Yamada, K.; Mito, F.; Matsuoka, Y.; Ide, S.; Shikimachi, K.; Fujiki, A.; Kusakabe, D.; Ishida, Y.; Enoki, M.; Tada, A.; Ariyoshi, M.; Yamasaki, T.; Yamato, M., Fluorescence probes to detect lipid-derived radicals. Nat. Chem. Biol. 2016, 12 (8), 608-613.

(2) Bligh, E. G.; Dyer, W. J., A rapid method of total lipid extraction and purification. Can. J. Biochem. Physiol. 1959, 37 (8), 911-917. 